\title{
Effect of oromotor stimulation on feeding performance and weight gain of preterm babies in selected hospital, Kolkata
}

\author{
Ankita Nath $^{1 *}$, Anuradha Mukherjee ${ }^{2}$, Supa Guha $^{3}$ \\ ${ }^{1}$ Sister Tutor, ${ }^{2}$ Senior Lecturer, ${ }^{3}$ Clinical Instructor, School of Nursing, I.P.G.M.E \& R,S.S.K.M. Hospital, Kolkata, ${ }^{2,3}$ West \\ Bengal Government College of Nursing, Kolkata, West Bengal, India
}

\section{*Corresponding Author: Ankita Nath}

Email: ankita.nath5@gmail.com

\begin{abstract}
Assessment of effect of oromotor stimulation on feeding performance and weight gain in selected hospitals, Kolkata.

Introduction: According to WHO, every year about 15 million babies are born prematurely around the world. Almost 1 million children die each year due to complication of preterm birth (2013). In India, out of 27 million babies born every year (2010 data), 3.5 million babies are premature.

Oral feeding difficulties are almost common in preterm neonates. Coordinated suck-swallow-breath pattern is not developed under 34 weeks of gestational age. So most of the preterm neonates are not able to have an independent feeding in that due time.

Aims: 1. To assess the effect of oromotor stimulation in the interventional group. 2. To compare the effect of oromotor stimulation between interventional group and the control group.

Design: Non probability purposive sampling technique was used to collect data from sixty preterm babies (30 in interventional group and 30 in control group) with the help of standardized IBFAT ( Infant Breast Feeding assessment tool) \& weight checking proforma. Inter rater reliability for IBFAT $(r=0.92)$ \& Weighing machine used for weight checking $(\mathrm{r}=0.93)$ was done. Pretest posttest time series design was adopted. Demographic data (gestational age , birth weight, age of day of $1^{\text {st }}$ intervention) was collected from each baby.

Results: Results revealed the significant difference in pre and post interventional breast feeding performance and weight of preterm babies between day 1 and day 7 in the experimental group as computed ' $t$ ' value 30.91[ (df 29) $=2.71, \mathrm{p}<0.001]$ and $11.87[$ ( df 29) $=2.71, \mathrm{p}<0.001]$ respectively. Result also showed significant difference in feeding performance and weight in experimental and the control group on day 7 as ' $t$ ' (df 58) $=9.3, p<0.001$ and 't' $($ df 58$)=2.23, \mathrm{p}<0.5$.

Conclusion: Oromotor stimulation is effective in improving feeding performance and weight gain of preterm baby.

Implication: In practice, oromotor stimulation can be practiced by nurse. The mothers of those preterm babies also can be taught this oromotor stimulation for practicing this method. In education, students may be provided with learning experiences in neonatal units and practice under supervision. Nurse administrator may develop protocol so that staff can practice independently in neonatal units. Research in this area of feeding performance improvement of preterm babies in the light of new intervention in the clinical field and utilization of this method (oromotor stimulation).
\end{abstract}

Keywords: Oromotor Stimulation, Feeding performance, Weight gain. 